\title{
Processing adds to the complexity
}

A recent study reveals how some of the increasingly apparent complexity of mammalian transcriptomes arises. It identifies an unanticipated abundance of stable, capped small RNAs in mammalian cells, which seem to be processed from both proteincoding and non-coding RNAs.

Deep sequencing identified 102,159 new small RNAs less than 200 nucleotides long from two

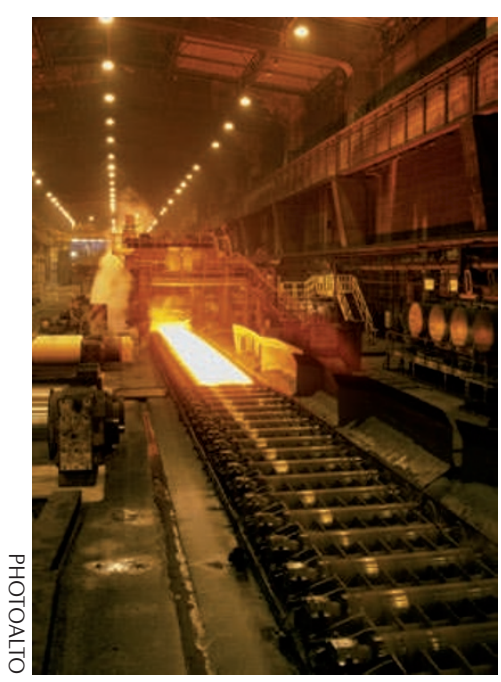

human cell lines. Many of these RNAs can be categorized as members of a previously identified class of small RNAs that lie no more than 500 nucleotides from a transcriptional start site (TSS) - so-called promoter-associated small RNAs (PASRs). The authors showed by enzymatic treatment and immunoprecipitation that PASRs have $5^{\prime}$ cap structures. There was also a correlation between many long RNA 5' ends that have been previously identified by CAGE tagging (which relies on the presence of a $5^{\prime}$ cap) and the 5' ends of the new small RNAs.

Both small RNAs and CAGE tags are particularly abundant in first exons, and the authors showed that many CAGE tags actually extend across splice sites, which suggests that they arise from the processing of spliced mRNAs, and then acquire a cap - a previously unrecognized fate for mRNAs. These long RNAs might then be further processed to generate smaller capped transcripts. The more detailed mapping of CAGE tags and small RNAs in one human gene strongly supported this conclusion.
These results suggest the regulated generation of abundant short RNAs in many mammalian protein-coding genes - but do these RNAs have any function? The authors made a series of synthetic single-stranded RNAs, 30-35 nucleotides in length, to mimic PASRs that lie near the annotated TSS of the human MYC gene. When these RNAs were transfected into cells, the result was a reduction in levels of MYC mRNA. This study has therefore not only revealed a new pathway of mRNA processing, but has also highlighted a potentially widespread transcriptional regulatory mechanism.

Louisa Flintoft

ORIGINAL RESEARCH PAPER Affymetrix/Cold Spring Harbor Laboratory ENCODE Transcriptome Project. Post-transcriptional processing generates a diversity of $5^{\prime}$-modified long and short RNAs. Nature 25 Jan 2009 (doi:10.1038/ nature07759)

FURTHER READING Wang, Z. et al. RNA-Seq: a revolutionary tool for transcriptomics. Nature Rev. Genet. 10, 57-63 (2009) | Kapranov, P. et al. Genome-wide transcription and the implications for genomic organization. Nature Rev. Genet. 8, 413-423 (2007) 